\title{
The usefulness of SPECT/CT in characterization of skeletal and soft tissue lesions - report of two cases
}

\author{
Marcin Pachowicz', ${ }^{1}$, Grzegorz Staśkiewicz ${ }^{2,}{ }^{3}$, Krzysztof Florek ${ }^{4}$ Beata E. Chrapko \\ ${ }^{1}$ Chair and Department of Nuclear Medicine, Medical University of Lublin, Poland \\ ${ }^{2}$ Chair and Department of Radiology, Medical University of Lublin, Poland \\ ${ }^{3}$ Chair and Department of Human Anatomy, Medical University of Lublin, Poland \\ ${ }^{4}$ St. John's Cancer Center, Lublin, Poland
}

[Received 29 V 2013; Accepted 7 I 2014]

\begin{abstract}
SPECT/CT imaging provides detailed information on the radiotracer distribution and enables simultaneous lesion morphology evaluation.

This hybrid imaging delivers complementary information about patient's disease.

We present two cases in which SPECT/CT imaging and cooperation between the nuclear medicine physician and radiologist quickly clarified the diagnosis, sparing patient unnecessary diagnostic procedures or treatment.
\end{abstract}

KEY words: SPECT, multimodality, spondylodiscitis, tuberculosis, goiter

Nuclear Med Rev 2014; 17, 1: 29-34

\section{Introduction}

SPECT/CT studies may be useful in the determination of some lesions of uncertain character in SPECT or CT alone.

We will present two cases in which hybrid imaging was a key to proper diagnosis.

SPECT/CT studies were performed using gamma camera Symbia T16 (Siemens, Erlangen, Germany). Diagnostic CT was applied (150 mAs)

\section{Presentation of cases}

\section{Case 1}

A 79-year old female patient with a history of osteoporosis and compressive fractures of vertebrae was admitted to our department due to severe back pain and a suspicion of malignancy.

The whole-body bone scan was performed 3 hours after the intravenous injection of $740 \mathrm{MBq}$ of $99 \mathrm{mTC}-\mathrm{MDP}$. It revealed high radiotracer accumulation in the lower part of the thoracic spine, involving most probably Th7/Th8 or Th8/Th9 vertebrae (Figure 1) and a small increased uptake area in one of the right ribs.

Correspondence to: Marcin Pachowicz MD

Chair and Department of Nuclear Medicine, Medical University of Lublin

8c Jaczewskiego St. 20-954 Lublin, Poland

Phone/fax: +48 817244339

E-mail: marcin.pachowicz.md@gmail.com
Chest SPECT study was performed. The data was collected in $128 \times 128$ matrix, 64 views, 15 seconds per view. Additionally x-ray computed tomography limited to the mid and lower chest was done.

Fused SPECT/CT images depicted the MDP accumulation mainly in the bodies and pedicles of two adjacent vertebrae (Figures $2 \mathrm{~A}$ and $3 \mathrm{~A}$ ). CT showed nearly total destruction of the end plates with spinal deformity (Figure $2 \mathrm{~B}$ and $3 \mathrm{~B}$ ). CT scans were suggestive of a spondylodiscitis rather than a lytic tumor. The tuberculous etiology should be included in the differential diagnosis and it was suggested independently by two radiologists: by one who evaluated MRI study performed prior to the bone scan study and the second one who read SPECT/CT.

The increased MDP uptake in the $8^{\text {th }}$ right rib was accompanied by focal osteosclerosis most probably associated with trauma.

The inflammation markers (CRP and ESR) level was significantly elevated. The WBC was mildly increased. On the other hand cancer markers including AFP, CEA, CA19-9 and CA-125 were negative.

Eventually, an active infectious process of the spine was excluded so no further actions were taken.

\section{Case 2}

A 69-year old female patient with a history of strumectomy was referred to our department with a recurrent goiter diagnosed by the ultrasound. Additionally, a tumor located in the upper mediastinum was previously detected by CT. Clinically, the patient was in euthyreosis. 


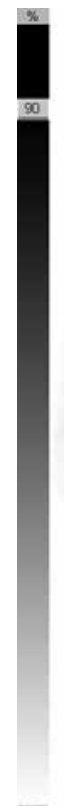

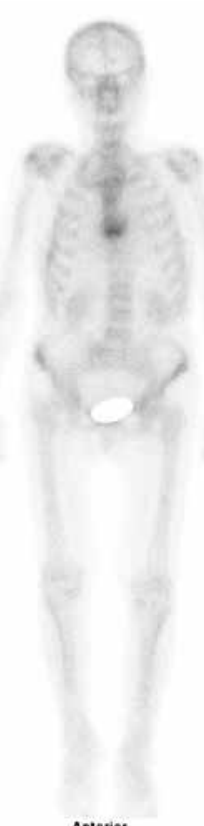

Anterior

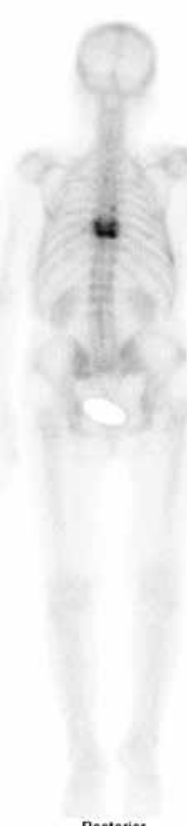

Posterio
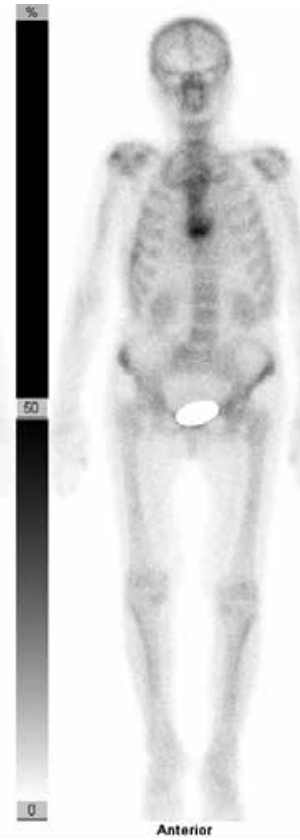

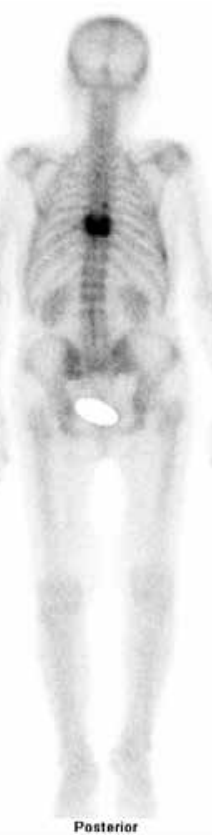

Figure 1. The whole body bone scan 3 hours after the i.v. administration of $740 \mathrm{MBq}$ of $99 \mathrm{mTc}$-MDP. An increased radiotracer uptake in the vertebrae of the lower thoracic spine, most probably Th7/Th8 or Th8/Th9 (difficult to distinguish on planar WB scan, especially in case of vertebral collapse). Additionally a small area of increased uptake is seen in one of the right ribs

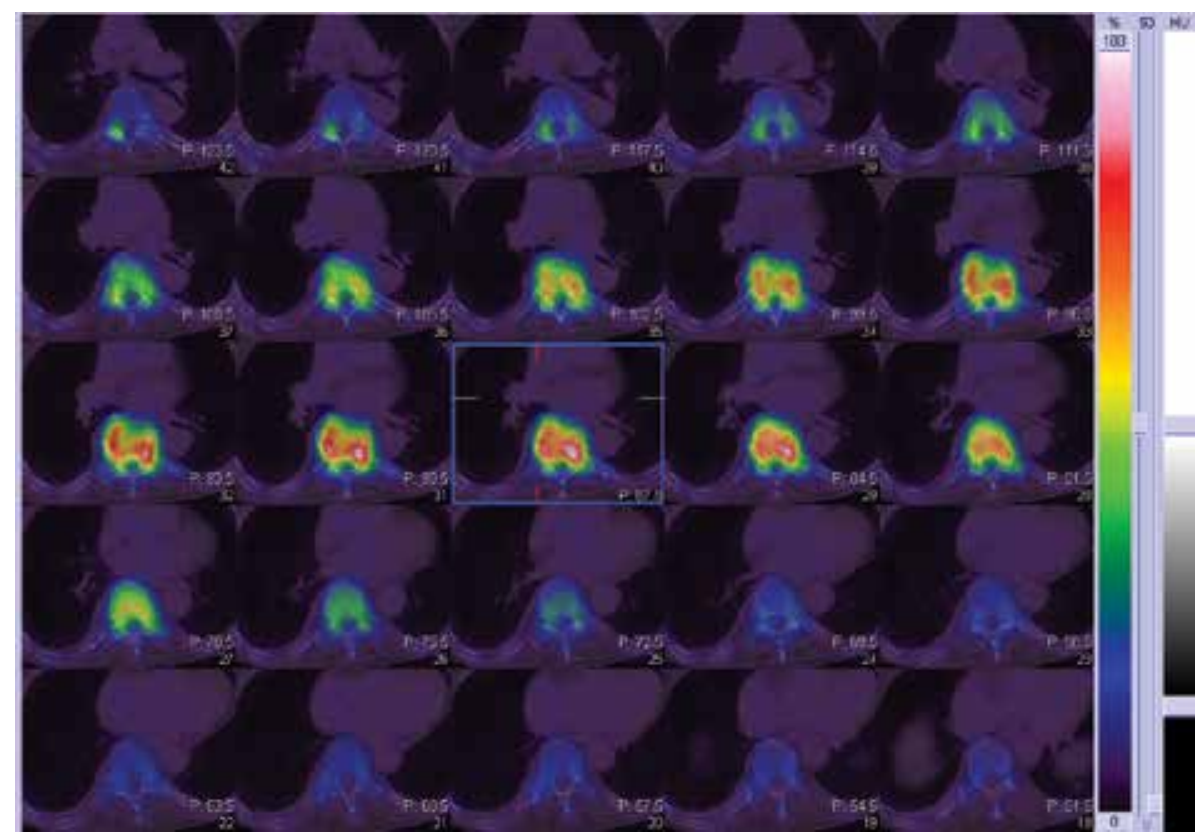

Figure 2A. SPECT/CT transverse sections indicate the radiotracer accumulation mainly in the bodies and pedicles of two adjacent vertebrae

The study was performed 30 minutes after intravenous administration of $111 \mathrm{MBq}$ of sodium pertechnetate. The first study was performed using a thyroid-dedicated gamma camera, then the second one with a large field of view gamma camera covering the area from the base of the skull to the epigastric region. Both scans presented a recurrent goiter and an abnormal area of the increased radiotracer uptake located in the upper mediastinum region (Figure 4).
Thereafter SPECT/CT study was performed over the lower neck and upper mediastinum (matrix $128 \times 128,64$ views, 30 seconds per view).

Evaluation of fused SPECT/CT images clearly indicated radiotracer accumulation within the clinically known tumor located anteriorly to the aortic arch (Figure 5A and 6A). The lesion itself was polycyclic in shape, slightly denser compared to muscles and with several hyperdense foci suggesting calcifications (Figure 5B and 6B). 


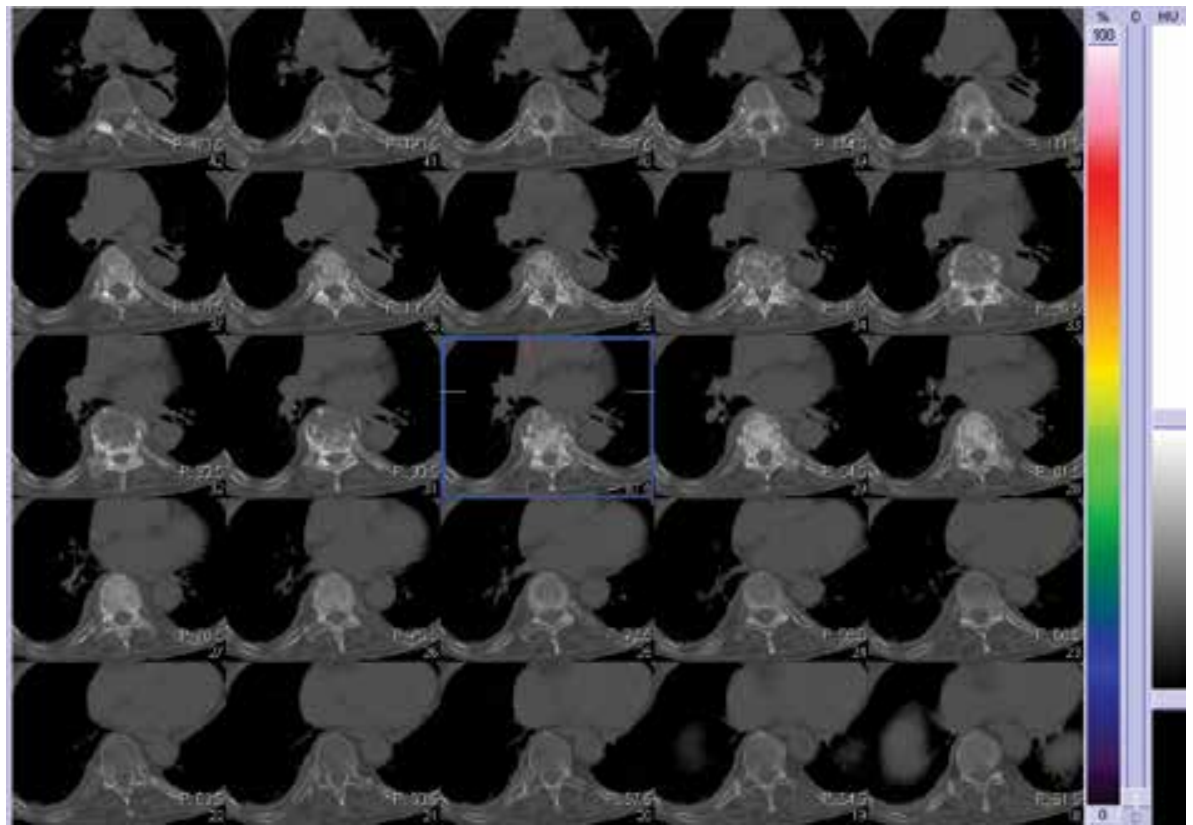

Figure 2B. CT transverse sections same as in Figure 2A show nearly total vertebral bodies' destruction ("exploded bodies") with high ${ }^{99 m T c-M D P}$ accumulation

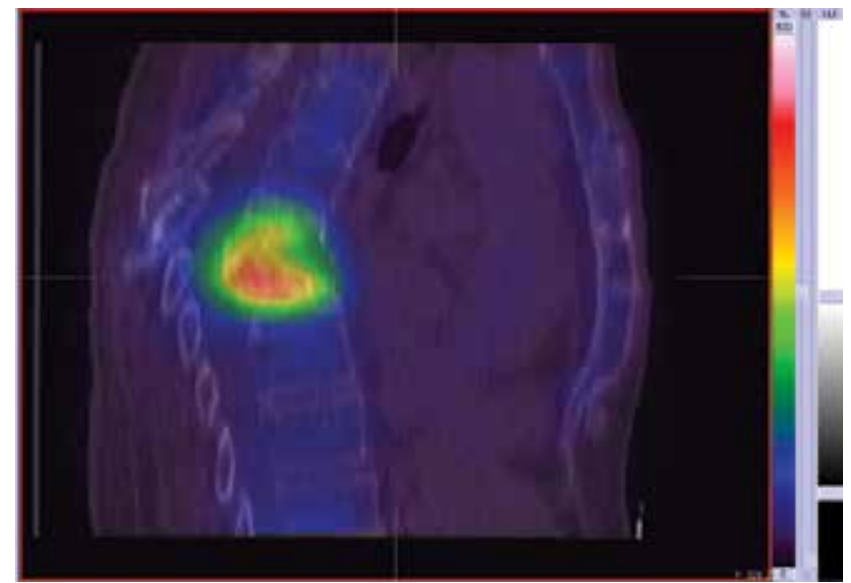

Figure 3A. SPECT/CT midline sagittal section. Increased uptake in the two adjacent vertebrae

All these findings confirmed thyroid origin of this tissue mass along with recurrent goiter in typical location.

\section{Discussion}

\section{Case 1}

The SPECT/CT role in clinical diagnosis is increasing, especially in bone scan. Planar images do not provide sufficient information about the exact location of abnormal radiotracer uptake in the vertebrae, but this can be obtained by SPECT technique. Additional CT study provides information about lesion morphology and, even more detailed, location. Lesions which affect body and pedicle or are photo-deficient are more likely to be malignant. Lesions affecting facet joints, costovertebral joints or two adjacent vertebrae are more likely to be benign [1-3].

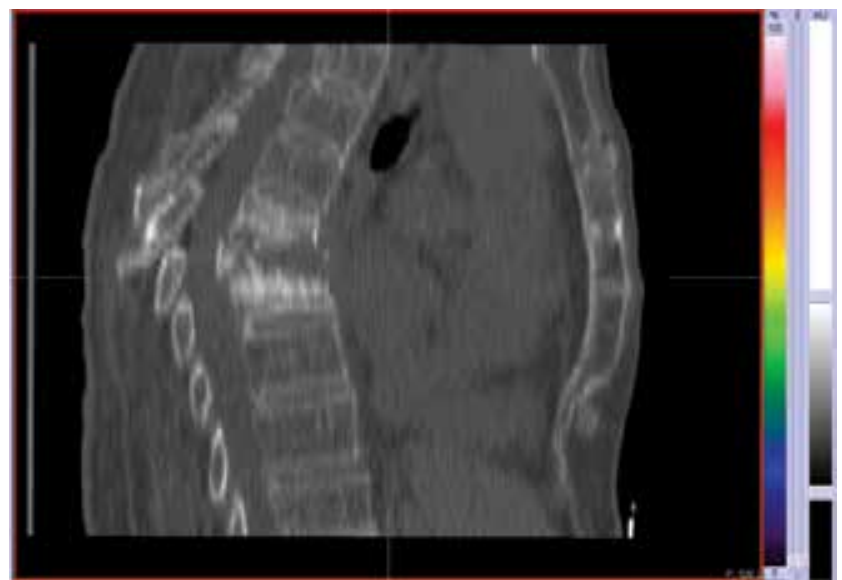

Figure 3B. CT midline sagittal section the same as in Figure 3A. Typical CT pattern of vertebral spondylodiscitis strongly suggesting tuberculosis. In such cases the infection starts in the anterior part of the vertebral body near end plate then spreads to destroy the entire body and intervertebral disc [5]

For characterizing vertebral lesion ${ }^{99 m} \mathrm{TC}-\mathrm{MDP}$ SPECT/CT is superior to planar bone scan and SPECT alone, but not to CT alone [4]. SPECT/CT also significantly reduces number of spinal lesion judged equivocal in SPECT alone [5].

Among all causes of the spondylodiscitis, the tuberculosis should always be kept in mind. It is estimated that even one-third of the world population is infected by Mycobacterium tuberculosis. There are nearly 10 million new cases each year including nearly half million of multi-drug resistant infection [6]. Although only 1 to $3 \%$ of patients with TB have the skeletal form, the spinal lesion may be the first manifestation of this disease [7]. 50\% of skeletal TB involves the spine and most commonly affects lower thoracic 
and upper lumbar region. The most common clinical manifestation is back pain, but the condition may be completely asymptomatic. In adults the vertebral body is the primary location. Posterior parts of the vertebrae are affected less frequently, but such condition has important implications on surgical management.

Differential diagnosis includes: metastatic disease or primary malignancy, fungal infection, pyogenic spondylitis, Langerhans cell histiocytosis, sarcoidosis or trauma [8].

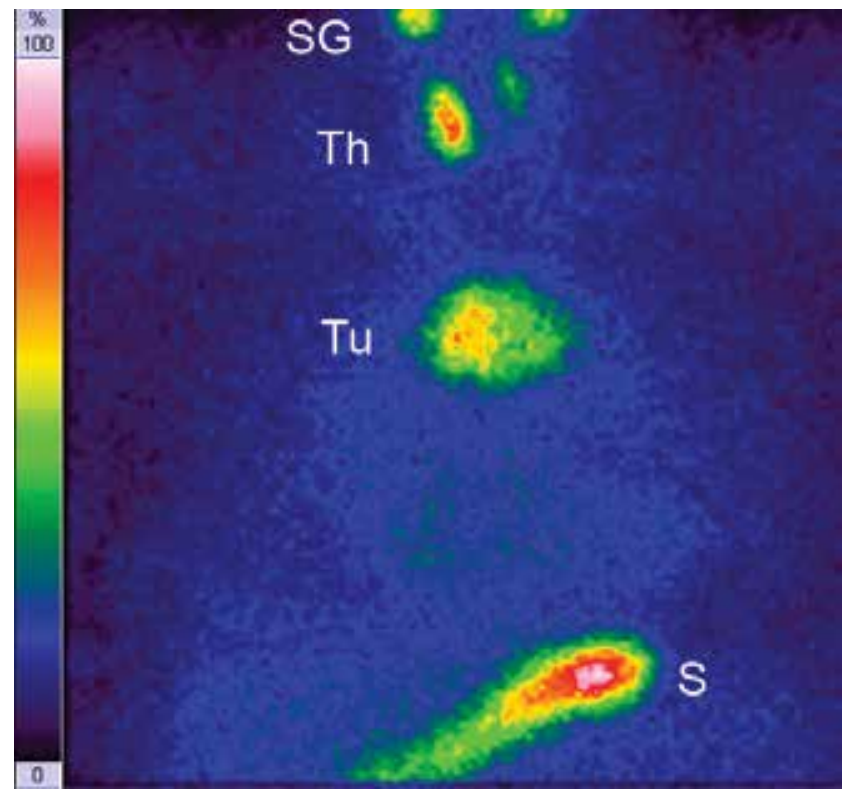

Figure 4. Anterior planar image of the neck and chest after intravenous administration of $111 \mathrm{MBq}$ of sodium pertechnetate. SG - salivary glands, Th - thyroid gland and S - stomach represent physiological distribution of the radiotracer. Tu - tumor in the upper mediastinum
The bone scan sensitivity for detection of skeletal tuberculosis is about $75 \%$ for spinal lesions only and over $90 \%$ for whole skeleton. Unfortunately, the specificity is low [9].

\section{Case 2}

Differential diagnosis of anterior mediastinum masses includes retrosternal goiter, lymphadenopathy, thymic lesions, germ cell tumors or even hemangiomas [10].

Sodium pertechnetate is the most widely used radiotracer utilized for evaluation of thyroid tissue. It is transported by the sodium-iodine symporter and its accumulation reflects function of follicular thyroid cells. After intravenous administration, it is additionally uptaken by salivary glands or gastric mucosa [11]. In case 2 correlation of scintigraphic and CT findings enabled easy recognition of the retrosternal mass character.

Mediastinal goiter after thyroidectomy is called "forgotten goiter" and is believed to be a very rare disease resulting from non-complete removal of plunging gland. Sometimes the mediastinal goiter may not be in connection with the normally located gland, what makes the diagnosis and surgery even more difficult [12].

The detailed goiter evaluation (size and location) before surgery is essential.

Considering all these facts it is also important to remember that there can be one limitation for such studies. The goiter may consist of tissue which does not uptake the pertechnetate (scintigraphically "cold") what could make SPECT/CT study useless. On the other hand, this "cold" goiter character (when suspected) may indicate malignancy, so we still obtain important information.

\section{Conclusions}

Multimodality imaging should be used widely and easily to gather additional information necessary for proper study interpreta-

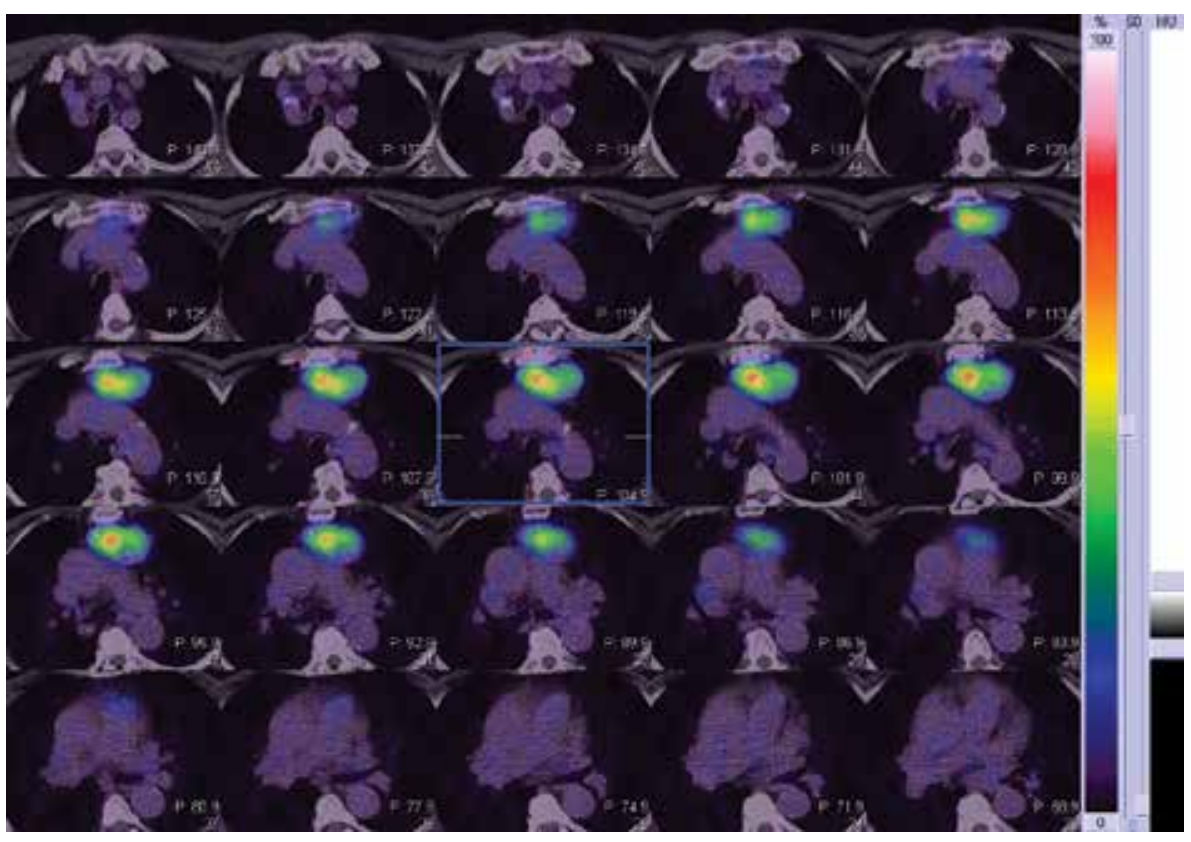

Figure 5A. SPECT/CT transverse images clearly indicate the pertechnetate accumulation in the tissue mass located between the aortic arch, left brachiocephalic vein and manubrium of the sternum 


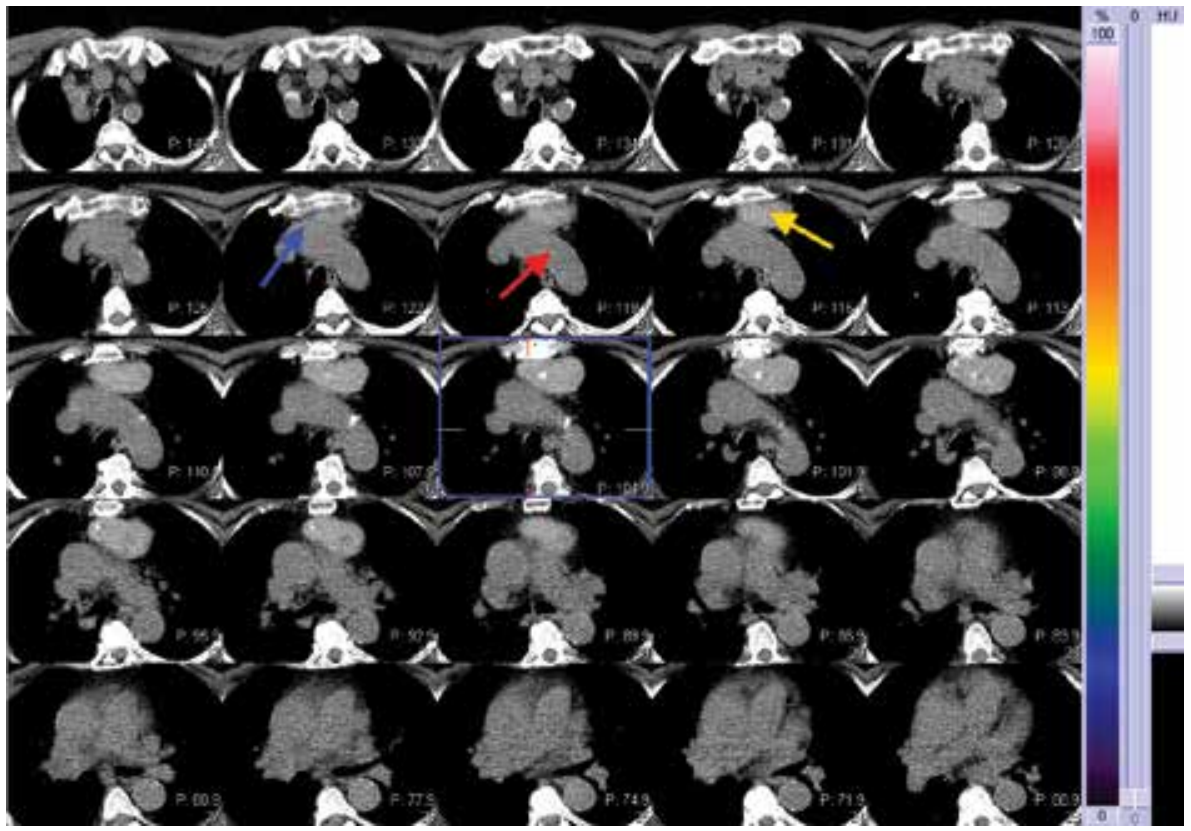

Figure 5B. CT transverse images the same as in Figure 5A. Red arrow - aortic arch, blue arrow — left brachiocephalic vein, yellow arrow tumor. Note polycyclic shape and hyperdense foci in the tumor

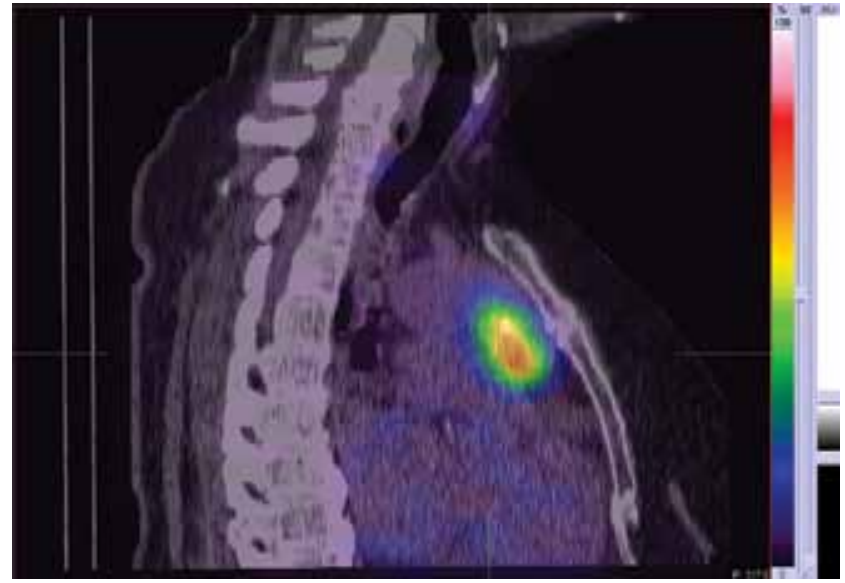

Figure 6A. SPECT/CT sagittal sections. The radiotracer uptake in anterior mediastinum

tion. As these two cases show, the crucial information may come both from CT (structural anomalies in case 1), or from SPECT (metabolic behavior of a tumor in case 2). The application of the SPECT/CT eliminates the necessity of subsequent verification and expedites the diagnosis.

Further development of hybrid techniques will surely increase the number of indications for such studies and will provide more information on clinical applications and proper interpretation.

\section{Abbervations}

AFP — alpha-fetoprotein

CEA - carcinoembrionic antigen

CA19-9 - cancer antigen 19-9

CA125 - cancer antigen 125

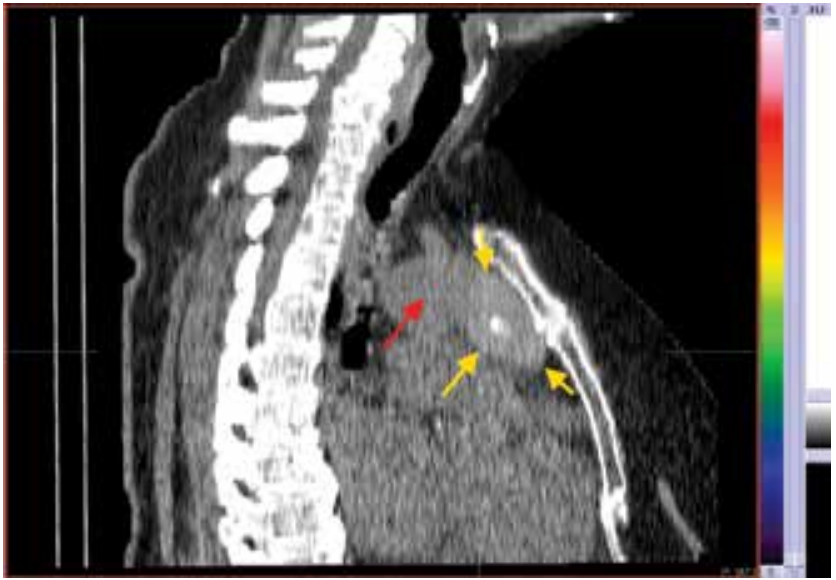

Figure 6B. CT sagittal sections. Arrows like in Figure 5B

CRP - C-reactive protein

CT - computed tomography

ESR - erythrocyte sedimentation rate

MDP — methylene diphosphonate

$\mathrm{MRI}$ - magnetic resonance imaging

SPECT — single photon emission computed tomography

TB - tuberculosis

\section{References}

1. Papathanassiou D, Bruna-Muraille C, Jouannaud C, Gagneux-Lemoussu L, Eschard JP, Liehn JC. Single-photon emission computed tomography combined with computed tomography (SPECT/CT) in bone diseases. Joint Bone Spine 2009; 76: 474-480.

2. Iqbal B, Currie GM, Wheat JM, Raza $\mathrm{H}$, Kiat $\mathrm{H}$. The incremental value of SPECT/CT in characterizing solitary spine lesions. J Nucl Med Technol 2011; 39: 201-207. 
3. Gnanasegaran G, Barwick T, Milburn H, Vijayanathan S, Fogelman I. Tuberculosis of the spine on Tc-99m MDP bone scan additional role of SPECT-CT. Clin Nucl Med 2009; 34: 271-274.

4. Sharma P, Dhull VS, Reddy RM, Bal C, Thulkar S, Malhotra A, Kumar R. Hybrid SPECT-CT for characterizing isolated vertebral lesions observed by bone scintigraphy: comparison with planar scintigraphy, SPECT, and CT. Diagn Interv Radiol 2013; 19: 33-40.

5. Zhang $\mathrm{Y}, \mathrm{Shi} \mathrm{H}, \mathrm{Gu} \mathrm{Y}$ et al. Differential diagnostic value of single-photon emission computed tomography/spiral computed tomography with Tc-99m-methylene diphosphonate in patients with spinal lesions. Nucl Med Commun 2011; 32: 1194-1200.

6. de la Vía E, Barón R, Molins E, Arriero JM. Tuberculosis. Arch Bronconeumol 2011; 47 (Suppl 8): 10-14.

7. Garg RK, Somvanshi DS. Spinal tuberculosis: a review. J Spinal Cord Med 2011; 34: 440-454.
8. Andronikou S, Bindapersad M, Govender N et al. Musculoskeletal tuberculosis - imaging using low-end and advanced modalities for developing and developed countries Tuberculosis of the Spine on Tc-99m MDP bone scan additional role of SPECT-CT. Acta Radiol 2011; 52: 430-441.

9. $\quad$ Lin WY, Wang SJ, Cheng KY, Shen YY, Changlai SP. Diagnostic value of bone and Ga-67 imaging in skeletal tuberculosis. Clin Nucl Med 1998 23: 743-746.

10. Whitten CR, Khan S, Munneke GJ, Grubnic S. A diagnostic approach to mediastinal abnormalities. Radiographics 2007; 27: 657-671.

11. Czepczyński R. Nuclear medicine in the diagnosis of benign thyroid diseases. Nucl Med Rev Cent East Eur 2012; 15: 113-119.

12. Sahbaz A, Aksakal N, Ozcinar B, Onuray F, Caglayan K, Erbil Y. The "forgotten" goiter after total thyroidectomy. Int J Surg Case Rep 2013; 4: 269-271. 